\title{
The Protective Effect of Cordycepin On Alcohol-Induced Osteonecrosis of the Femoral Head
}

\author{
Yi-Xuan Chen ${ }^{a}$ Dao-Yu Zhu ${ }^{a}$ Zheng-Liang Xua Jun-Hui Yin ${ }^{b} \quad$ Xiao-Wei Yu \\ Jiong Meia You-Shui Gao Chang-Qing Zhanga,b \\ aDepartment of Orthopedic Surgery, Shanghai Jiao Tong University Affiliated Sixth People's Hospital, \\ Shanghai, 'Institute of Microsurgery on Extremities, Shanghai Jiao Tong University Affiliated Sixth \\ People's Hospital, Shanghai, China
}

\section{Key Words}

Cordycepin • Osteonecrosis of the femoral head • Alcohol • Osteogenesis • Wnt/ $\beta$-catenin pathway $\cdot$ Human bone mesenchymal stem cells

\begin{abstract}
Background: Alcohol abuse is known to be a leading risk factor for atraumatic osteonecrosis of the femoral head (ONFH), in which the suppression of osteogenesis plays a critical role. Cordycepin benefits bone metabolism; however, there has been no study to determine its effect on osteonecrosis. Methods: Human bone mesenchymal stem cells (hBMSCs) were identified by multi-lineage differentiation. Alkaline phosphatase (ALP) activity, RT-PCR, western blots, immunofluorescent assay and Alizarin red staining of BMSCs were evaluated. A rat model of alcohol-induced ONFH was established to investigate the protective role of cordycepin against ethanol. Hematoxylin \& eosin (H\&E) staining and micro-computerized tomography (micro-CT) were performed to observe ONFH. Apoptosis was assessed by TdT-mediated dUTP nick end labeling (TUNEL). Immunohistochemical staining was carried out to detect OCN and COL1. Results: Ethanol significantly suppressed ALP activity, decreased gene expression of OCN and BMP2, lowered levels of RUNX2 protein, and reduced immunofluorescence staining of OCN and COL1 and calcium formation of hBMSCs. However, these inhibitory effects were attenuated by cordycepin co-treatment at concentrations of 1 and $10 \mu \mathrm{g} / \mathrm{mL}$. Moreover, it was revealed that the osteo-protective effect of cordycepin was associated with modulation of the Wnt/ $\beta$-catenin pathway. In vivo, by micro-CT, TUNEL and immunohistochemical staining of OCN and COL1, we found that cordycepin administration prevented alcohol-induced ONFH. Conclusion: Cordycepin treatment to enhance osteogenesis may be considered a potential therapeutic approach to prevent the development of alcohol-induced ONFH.
\end{abstract}

Y.X. Chen and D.Y. Zhu contributed equally to the manuscript.

You-Shui Gao, Ph.D., M.D.

and Chang-Qing Zhang, Ph.D., M.D.
Department of Orthopedic Surgery, Shanghai Jiao Tong University Affiliated Sixth People's Hospital, 600 Yishan Road, Shanghai, E-mail: gaoyoushui@163.com; E-mail: zhangcq@sjtu.edu.cn

\section{KARGER}




\section{Cellular Physiology Cell Physiol Biochem 2017;42:2391-2403

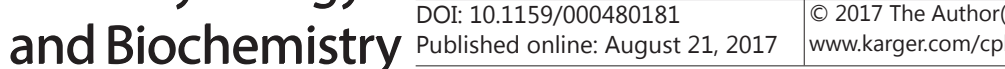 \\ Chen et al.: Protective Effect of Cordycepin On Alcohol-Induced ONFH}

\section{Introduction}

Epidemiologic studies indicate that long-term and high-dose consumption of alcohol is a leading risk factor of osteonecrosis of the femoral head (ONFH). Other causes include steroids, trauma and hyperlipidemia [1-5]. The exact physiological mechanisms underlying alcohol-induced ONFH remain unclear. The current study aimed to investigate a potential strategy to halt the progression of alcohol-induced ONFH.

Alcoholics showed characteristics of decreased bone synthesis, increased bone resorption and lower bone mineral density (BMD) [1, 6-8]. Animal studies also indicated decreased osteogenesis and bone volume after long-term alcohol consumption $[9,10]$. Human bone mesenchymal stem cells (hBMSCs) are regarded as the main source of selfrenewal and regeneration of bone tissue [11-13], whereas recent studies demonstrated that ethanol treatment could impair the osteogenic differentiation of hBMSCs [2, 14]. Meanwhile, suppressed osteogenic activity has been detected in ONFH patients [15]. Restoration of osteogenic potential could alleviate the progression of ONFH in animal experiments [16]. Hence, we mainly focused on the rescue of ethanol-induced anti-osteogenic effects to reduce the development of alcohol-induced ONFH.

Cordycepin is one of the major bioactive components of cordyceps militaris, a valued traditional Chinese medicine [17]. Cordycepin has a variety of biological functions, including anti-tumor, anti-viral, anti-oxidant, and anti-inflammatory properties [17-19]. A recent study demonstrated the osteo-protective effects of cordycepin by enhancing the expression of osteogenesis-related genes in BMSCs [20]. Currently, there is no report regarding the application of cordycepin to alcohol-induced ONFH. We hypothesized that cordycepin might exert protective effects against alcohol-induced ONFH.

Wnt/ $\beta$-catenin signaling has been regarded as a fundamental signaling pathway in the determination of osteogenic differentiation, proliferation and migration. Activation of canonical Wnt signaling leads to enhanced bone mineral density, whereas disruption of its activation impairs osteogenesis. Previous studies indicated that ethanol inhibited osteogenic differentiation and unregulated the adipogenic differentiation of BMSCs partly via the suppression of Wnt/ $\beta$-catenin signaling [21]. Therefore, we investigated the effect of cordycepin on Wnt/ $\beta$-catenin signaling in ethanol-treated hBMSCs. For in vivo experiments, we established an alcohol-induced ONFH model with the Lieber-DeCarli liquid diet. This rat model is a well-known experimental model for investigating the pathological process and intervening strategies of alcohol-induced ONFH [22]. In vitro, a plethora of assays were employed to reveal that ethanol-induced anti-osteogenic effects were alleviated by cordycepin in hBMSCs. Functional studies indicated that the ethanol-induced suppression of Wnt/ $\beta$-catenin signaling was reversed by cordycepin. Hence, we demonstrate that the $W n t / \beta$-catenin pathway is associated with the suppression of osteogenic differentiation by ethanol in BMSCs, which contributes to the progression of alcohol-induced ONFH, while cordycepin may be considered a preservation strategy.

\section{Materials and Methods}

\section{The isolation of hBMSCs}

hBMSCs were harvested from patients undergoing hip arthroplasty due to femoral neck fractures. The hBMSCs were cultured with $\alpha$-MEM (Gibco, Shanghai, China) supplemented with 10\% FBS (Gibco, Shanghai, China), $1 \%$ penicillin and streptomycin (Gibco, Shanghai, China) and maintained at $37{ }^{\circ} \mathrm{C}$ with $5 \%$ $\mathrm{CO}_{2}$. The passage of hBMSCs was carried out when they reached a confluence of 70-80\%. The hBMSCs of six to nine passages were used in all experiments. This study was approved by the Institutional Ethics Review Committee at Shanghai Sixth People's Hospital (2010-20). All experiments on hBMSCs were performed according to approved guidelines and regulations. 


\section{Cellular Physiology Cell Physiol Biochem 2017;42:2391-2403

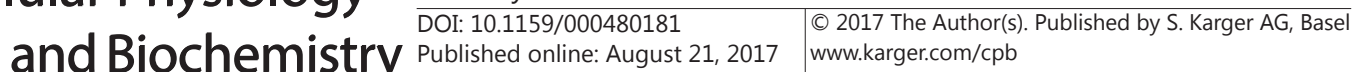

Chen et al.: Protective Effect of Cordycepin On Alcohol-Induced ONFH

\section{Cell toxicity and proliferation}

The effect of ethanol and cordycepin on the proliferation of BMSCs was assessed using a cell viability assay (Cell Counting Kit-8 (CCK-8); Dojindo Molecular Technologies, Inc., Japan). Briefly, BMSCs were seeded in 96-well plates at an initial density of $5 \times 10^{3}$ cells/well and cultured in $50 \mathrm{mM}$ of ethanol and/or different concentrations of cordycepin $(0.1 \mu \mathrm{g} / \mathrm{ml}, 1 \mu \mathrm{g} / \mathrm{ml}$ and $10 \mu \mathrm{g} / \mathrm{ml})$ for $1,3,5$ and 7 days. Then, $20 \mu \mathrm{l}$ of CCK- 8 solution and $180 \mu \mathrm{l}$ of culture medium were added to each well at each time point and incubated for 2 hours at $37^{\circ} \mathrm{C}$. Aliquots $(100 \mu \mathrm{l})$ were then taken from each well and transferred to another 96 -well plate. The absorbance of the samples was measured at $450 \mathrm{~nm}$ with a spectrophotometric microplate reader (Bio-Rad 680 , USA). The results were expressed as the optical density of the aliquots minus the absorbance of the blank wells.

\section{Induction of differentiation}

The potential of hBMSCs to differentiate into osteogenic, chondrogenic, and adipogenic cells was analyzed. A number of $2 \times 10^{5}$ of hBMSCs was seeded in a 6 -well plate. To induce osteogenic differentiation, cells were cultured with osteogenic differentiation media for 21 days (Life Technologies, Gibco). The calcium deposits were examined by Alizarin red. For chondrogenesis induction, cells were treated with chondrogenic differentiation media (Life Technologies, Gibco) for 21 days. Positive induction was detected by Toluidine blue staining after differentiation. For adipogenic induction, hBMSCs were cultured with adipogenic differentiation media for 21 days (Life Technologies, Gibco). The formation of lipid vacuoles was observed using Oil Red 0 staining. Images were captured with a LEICA DM 4000 microscope.

\section{ALP activity}

To measure ALP activity, $1 \times 10^{5}$ cells per well were seeded in 48 -well plates and grown to $90 \%$ confluence within 48 hours. The culture medium was then replaced with $\alpha$-MEM supplemented with $10 \% \mathrm{FBS}, 1 \%$ penicillin and streptomycin, $1 \mathrm{mM}$ of ascorbyl-2-phosphate, $4 \mathrm{mM}$ L-glutamine and 100 $\mathrm{U} / \mathrm{mL}$ each of penicillin and streptomycin (Gibco, Carlsbad, CA), which is the condition known to drive osteogenic differentiation. The osteogenic differentiation medium was refreshed every day. The ALP activity was measured at multiple time points ( 7 and 14 days) using a microplate test kit (Nanjing Jiancheng Bioengineering Institute, Nanjing, China)

\section{RT-PCR}

The gene expressions of BMP2 and OCN were measured using the qRT-PCR. Total mRNA was extracted using Trizol Reagent (Invitrogen). A reverse-transcription procedure was carried out using EasyScript OneStep gDNA Removal and cDNA Synthesis Supermix (TransGen Biotech, Beijing, China). The qRT-PCR was performed on ABI HT7900 (Applied Biosystems, Australia) using TransStart Tip Green qPCR SuperMix (TransGen Biotech) according to the manufacturer's instructions. The relative expression of the genes was normalized to $\beta$-actin. The forward and reverse primers were provided by BioTNT (BioTNT, Shanghai, China). OCN: forward: 5' AGC CTT TGT GTC CAA GCA-3' and reverse 5' CCA GCC ATT GAT ACA GGT AG-3'. BMP2: forward: 5' GCA GGT GGG AAA GTT TTG AT-3' and reverse 5' GCT GTT TGT GTT TGG CTT GA. $\beta$-actin, forward: 5' AAG GTG ACA GCA GTC GGT T-3' and reverse: 5'-TGT GTG GAC TTG GGA GAG G-3'. The relative mRNA expressions were normalized to the level of $\beta$-actin mRNA.

\section{Western blotting}

The proteins were obtained from hBMSCs using a cell lysis buffer supplemented with proteinase inhibitor. The amount of protein was measured using the BCA protein assay kit (Cell Signaling Technology, Danvers, MA). Next, $20 \mu \mathrm{g}$ of protein was resolved on SDS-PAGE gels and transferred to PVDF membranes. The protein was blocked with $5 \%$ milk in Tris-buffered saline $0.1 \%$ Tween (TBST). The membrane was then incubated with primary antibodies. Primary antibodies including anti- $\beta$-catenin, anti- $\beta$-actin and antiRunx2 were provided by Cell Signaling Technology. The membranes were then incubated with an anti-rabbit and anti-mouse secondary antibody (Cell Signaling Technology). After chemiluminescence, a LEICA DM 4000 was used to detect the target bands. The protein levels were normalized by $\beta$-actin. Quantification of protein was carried out using ImageJ Software. 


\section{Cellular Physiology Cell Physiol Biochem 2017;42:2391-2403

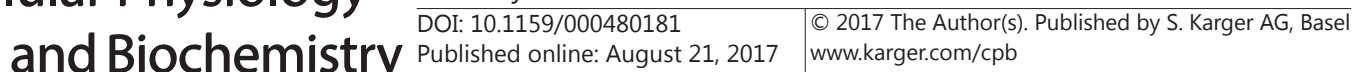

Chen et al.: Protective Effect of Cordycepin On Alcohol-Induced ONFH

\section{Immunofluorescent staining}

Cells were seeded on $0.1 \%$ gelatin-coated glass cover slips, placed in a 6-well plate and allowed 48 hours for adherence. Cells were then incubated with culture medium supplemented with ethanol (50 mM) and/or different concentrations of cordycepin as indicated. Cells were fixed by $4 \%$ paraformaldehyde for 20 min, permeabilized with $0.3 \%$ Triton X-100 in PBS for 15 min, and incubated with anti-OCN (Proteintech, Shanghai, China) and anti-COL1 (Proteintech, Shanghai, China) at $4{ }^{\circ} \mathrm{C}$ overnight. Cells were then rinsed and incubated with the Alexa fluor ${ }^{\mathrm{TM}} 488$ secondary antibody (Invitrogen). Visualization of cell nuclei and skeletons was performed by 4',6-diamidino-2-phenylindole (DAPI) and phalloidine. The immunofluorescence images were captured using an immunofluorescence microscope.

\section{Animal grouping and treatment}

Sprague-Dawley rats at 8-weeks of age were used for the animal model. All procedures were carried out with the approval of the Animal Research Committee at Shanghai Sixth People's Hospital. A total of 30 rats were randomly divided into three groups: (1) normal control (NC) group, (2) alcohol group (AL) group, and (3) alcohol + cordycepin group (AL+cordycepin) group. The rats in the AL group received an ethanol-containing Lieber-DeCarli liquid diet (FBSH, Shanghai, China), which contains 8\% (weight/volume) ethanol (36\% of daily calories), fat (38\% of daily calories) and protein ( $17 \%$ of daily calories) for 6 weeks. In addition, rats in the AL+cordycepin group received the same ethanol-containing diet for 6 weeks while being co-treated with cordycepin $(10 \mathrm{mg} / \mathrm{kg} / \mathrm{d}$ ) by intraperitoneal (i.p) injection [22, 23]. Control rats were fed with the Lieber-DeCarli diet without ethanol (the ethanol was substituted with maltodextrin, which provides the same amount of calories) for 6 weeks. All rats were allowed to adapt to the Lieber-DeCarli diet for one week prior to the start of experiment. All thirty rats had free access to the diets. Diets were prepared fresh daily.

\section{Micro-CT scanning}

All thirty left femoral heads were observed using micro-CT scanning. The micro-CT scanner was set at 9-micron voxel size. Image acquisition was carried out at $35 \mathrm{kV}$ of energy and $220 \mathrm{~mA}$ of intensity. The reconstruction of the image was carried out with CTVol software. The trabecular bone volume fraction (BV/ TV) and trabecular thickness (Tb.Th) were calculated based on reconstructed images.

\section{Hematoxylin \& eosin staining}

Decalcification of specimens was carried out with EDTA with constant shaking for one month. After decalcification, the bone tissue was embedded in paraffin and cut into 5-mm-thick sections. For the evaluation of subchondral trabecular structure, five specimens were stained with hematoxylin \& eosin (H\&E) and examined using a LEICA DM 4000 microscope to unveil the grand morphology of the femoral heads. Images were captured with a LEICA DM 4000 microscope.

\section{TUNEL}

For TdT-mediated dUTP nick end labeling (TUNEL), three specimens from each group were deparaffinized. The procedure was performed with a TUNEL staining kit (Roche) following the manufacturer's instructions. Images were captured with a LEICA DM 4000 microscope. The quantification of positive staining was analyzed with Image-Pro Plus Software.

\section{Immunofluorescence staining}

Three decalcified specimens from each group were deparaffinized; the antigens were retrieved and blocked with 1\% BSA and treated with anti-OCN (Proteintech, Shanghai, China) and anti-COL1 (Proteintech, Shanghai, China) primary antibodies for 2 hours at room temperature and then treated with a secondary antibody for 1 hour at room temperature. The 3, 30-diaminobenzidine (DAB) substrate was used to visualize the immune-reactivity. Images were acquired using a LEICA DM 4000 microscope and analyzed with ImagePro Plus Software. The mean density (IOD/area) was calculated and counted.

\section{Statistical analysis}

Values were reflected as the mean $\mathrm{s} \pm$ SE. SPSS 20.0 software was used to analyze the data in all groups. Comparisons among multiple groups were performed using a one-way analysis of variance (ANOVA) and 


\section{Cellular Physiology \\ Cell Physiol Biochem 2017;42:2391-2403 and Biochemistry

Bonferroni correction. $P$ values less than 0.05 were considered statistically significant.

\section{Results}

Multi-lineage differentiation of hBMSCs

The characteristics of the hBMSCs used in the following experiments were examined to determine their multi-lineage differentiation potential into osteogenic, chondrogenic, and adipogenic cells. Using the pellet test, we confirmed chondrogenic differentiation (Fig. 1A). The Alizarin red staining (ARS) revealed osteogenic differentiation (Fig. 1B), whereas adipogenic differentiation was verified using oil red 0 staining

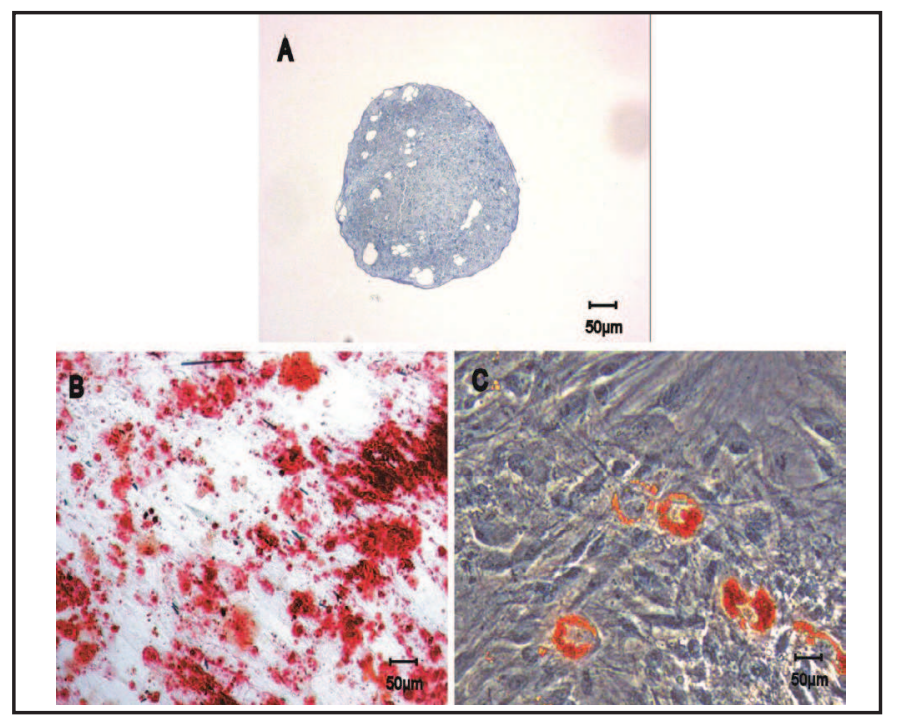

Fig. 1. Multi-lineage differentiation of hBMSCs. (A) Pellet test after 21 days of chondrogenic differentiation in hBMSCs. (B) Alizarin red staining of hBMSCs after 21-day incubation with osteogenic medium. (C) Oil red 0 staining showing adipogenic differentiation of hBMSCs after 21 days of incubation. (Fig. 1C).

Cordycepin alleviated the ethanol-induced inhibition on osteogenic differentiation of hBMSCs

Following treatment with ethanol and/or ethanol supplemented with different concentrations of cordycepin, ALP activity of hBMSCs was detected. Fig. 2A and Fig. 2B show that ethanol decreased the ALP activity at both 7 and 14 days, whereas supplementation of $1 \mu \mathrm{g} / \mathrm{ml}$ and $10 \mu \mathrm{g} / \mathrm{ml}$ cordycepin significantly reversed the inhibition effect $(p<0.05)$. However, no significant increase of ALP activity was detected at the level of $0.1 \mu \mathrm{g} / \mathrm{ml}$ cordycepin (Fig. 2A and Fig. 2B). We further detected the expression of osteogenesis-related markers of hBMSCs treated with ethanol alone and ethanol supplemented with cordycepin. The results of RT-PCR showed that the gene expression (BMP2 and OCN) of hBMSCs was markedly decreased when treated with ethanol at 24 hours (Fig. $2 \mathrm{C}$ and Fig. 2D, $p<0.05$ ). The supplementation of $0.1 \mu \mathrm{g} / \mathrm{ml}, 1 \mu \mathrm{g} / \mathrm{ml}$ and $10 \mu \mathrm{g} / \mathrm{ml}$ cordycepin significantly blocked the inhibition effect by ethanol on BMP2 gene expression (Fig. $2 \mathrm{C}, p<0.05$ ). The OCN gene expression was upregulated under additional cordycepin at the levels of $1 \mu \mathrm{g} / \mathrm{ml}$ and $10 \mu \mathrm{g} /$ $\mathrm{ml}$ (Fig. 2D, $p<0.05$ ).

The protein level of Runx2 was also detected. The results showed that $50 \mathrm{mM}$ ethanol treatment decreased the level of Runx2, whereas supplementation of $1 \mu \mathrm{g} / \mathrm{ml}$ and $10 \mu \mathrm{g} /$ $\mathrm{ml}$ cordycepin obviously enhanced Runx2 expression (Fig. 2E). To visualize the effect of ethanol and cordycepin on proteins of OCN and COL1, we carried out immunofluorescent staining. The hBMSCs exhibited significantly less staining of OCN and COL1 when treated with ethanol alone, while an obvious increase of both proteins was detected with additional $10 \mu \mathrm{g} / \mathrm{ml}$ cordycepin (Fig. 2G). To measure the effect of ethanol and cordycepin on hBMSCs proliferation, we carried out CCK-8 assay. We noted that the number of hBMSCs significantly increased after 5-day and 7-day incubation with ethanol compared to that in the control group (Fig. $2 \mathrm{~F}, p<0.05$ ). No significant difference in cell number was observed between each cordycepin-treated group and control group (Fig. 2 F, $p<0.05$ ). Finally, the result of ARS demonstrated that ethanol suppressed the osteogenic differentiation of hBMSCs. The mineralization of hBMSCs improved most significantly when co-treated with $10 \mu \mathrm{g} / \mathrm{ml}$ of cordycepin compared with that of other groups (Fig. 2H). 


\section{Cellular Physiology Cell Physiol Biochem 2017;42:2391-2403 \begin{tabular}{ll|l} 
and Biochemistry Published online: August 21, 2017 & $\begin{array}{l}\text { O } 2017 \text { The Author(s). Published by S. Karger AG, Basel } \\
\text { www.karger.com/cpb }\end{array}$ \\
\cline { 2 - 3 }
\end{tabular}}

Fig. 2. Cordycepin alleviated the ethanol-induced inhibition on osteogenesis of hBMSCs. (A-B) The ALP activity of hBMSCs was measured at 7 and 14 days. hBMSCs were cultured in osteogenic medium supplemented with ethanol and/or cordycepin as indicated. (Values are shown as the mean $\pm \mathrm{SE}(\mathrm{N}=3) * \mathrm{p}<0.05)$ (C-D) The mRNA expression of BMP2 and OCN in hBMSCs after 24 hours incubation of ethanol and/or cordycepin. (Values are shown as the mean $\left.\pm \mathrm{SE}(\mathrm{N}=3){ }^{*} \mathrm{p}<0.05\right)$. (E) hBMSCs were incubated for 72 hours. The RUNX2 level was decreased by ethanol and increased by cordycepin in hBMSCs. Proteins were immunoblotted with primary antibodies against RUNX2. $\beta$-actin served as a normalization control. (F) Proliferation of hBMSCs incubated for $1,3,5$ and 7 days in medium supplemented with $50 \mathrm{mM}$ ethanol and/or cordycepin as indicated. Cordycepin did not impair the survival and proliferation of hBMSCs. (G) hBMSCs were incubated with $50 \mathrm{mM}$ ethanol and/or 10 $\mu \mathrm{g} / \mathrm{ml}$ of cordycepin for 72 hours in osteogenic medium. Cells were immunostained with OCN and COL1. Cell skeleton was stained with phalloidine, and cell nuclei were stained with DAPI. (H)

The ethanol-induced anti-osteogenic effect in hBMSCs was reversed by cordycepin. Cells were incubated for 21 days with osteogenic medium and stained with Alizarin red.

The osteo-protective effects of cordycepin was associated with the Wnt/ $\beta$-catenin pathway

To probe into the underlying mechanism of protective effects of cordycepin, we carried out western blot of $\beta$-catenin, which is the center mediator of $\mathrm{Wnt} / \beta$-catenin signaling. The result showed significantly decreased $\beta$-catenin in hBMSCs treated by ethanol, whereas $1 \mu \mathrm{g} / \mathrm{ml}$ and $10 \mu \mathrm{g} / \mathrm{ml}$ cordycepin clearly alleviated this blockage effect (Fig. 3A). Quantification of the western blot result is shown in Fig. 3B $(p<0.05)$. 


\section{Cellular Physiology Cell Physiol Biochem 2017;42:2391-2403 \begin{tabular}{ll|l}
\hline DOI: 10.1159/000480181 & 2017 The Author(s). Published by S. Karger AG, Basel \\
\hline
\end{tabular} and Biochemistry Published onIne: August 21, 2017 www.karger.com/cpb}

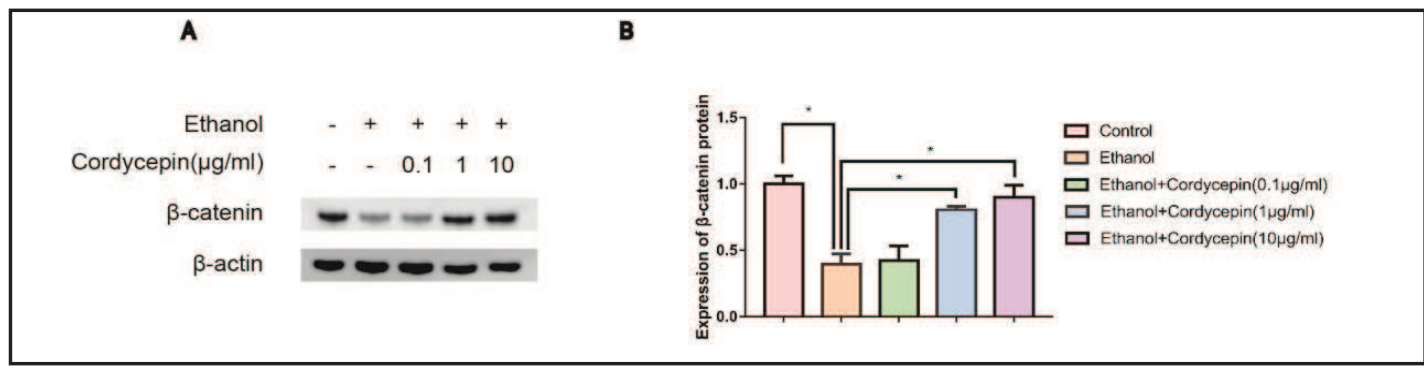

Fig. 3. The osteo-protective effects of cordycepin was associated with the Wnt/ $\beta$-catenin pathway. (A) $\beta$-catenin was decreased by $50 \mathrm{mM}$ ethanol treatment and increased by cordycepin. hBMSCs were incubated for 72 hours and immunoblotted for $\beta$-catenin. $\beta$-actin served as a normalization control. (B) Quantification of $\beta$-catenin. (Values are shown as the mean $\left.\pm \mathrm{SE}(\mathrm{N}=3),{ }^{*} \mathrm{p}<0.05\right)$.

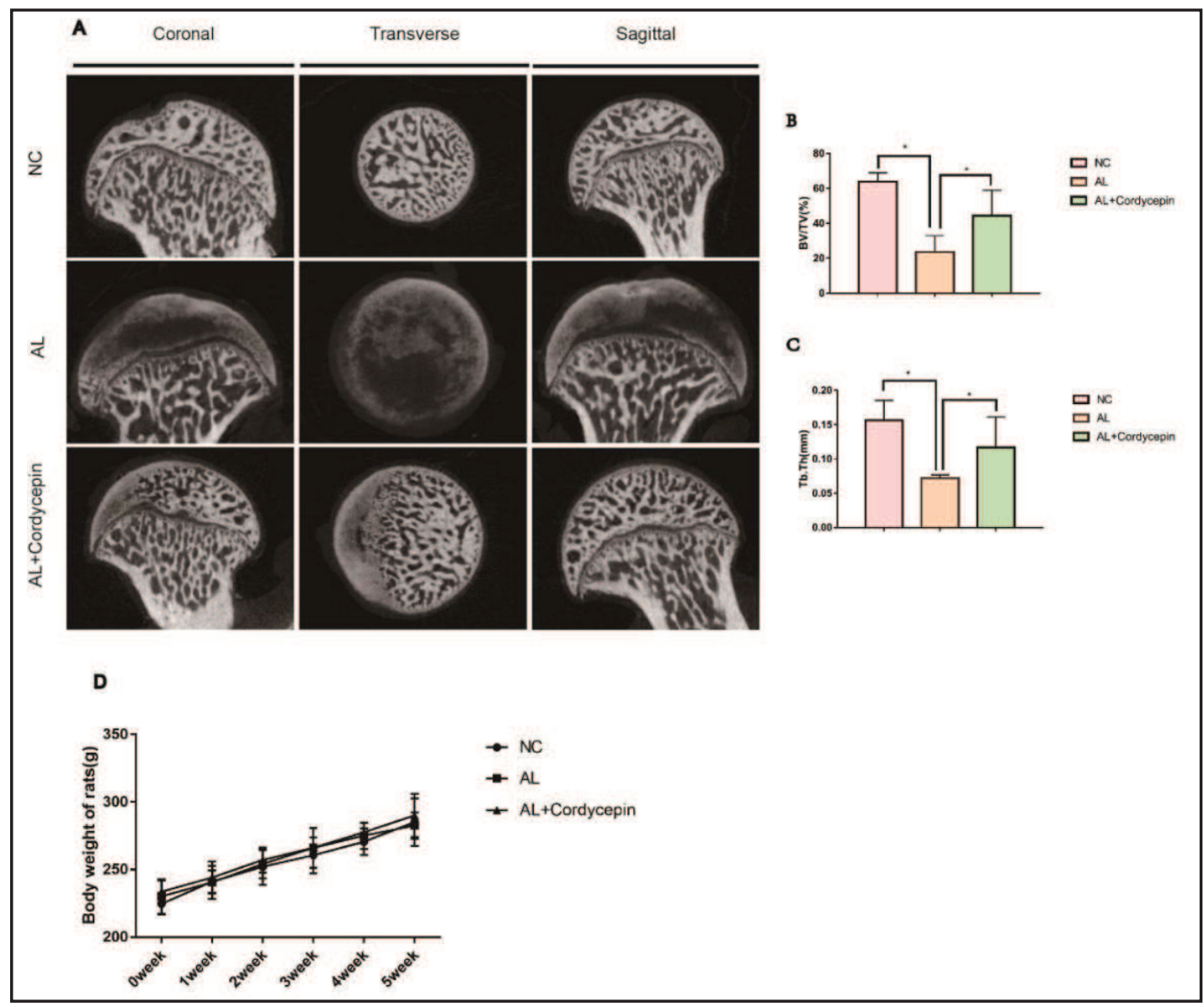

Fig. 4. Micro-CT analyses. (A) Micro-CT scanning images of the femoral head divided by group and section. Significantly less subchondral trabecular bone was observed in the AL group than in the NC group while more was observed in the AL+ cordycepin group. (B-C) BV/TV and Tb.Th were calculated based on reconstructed CT images. Values are represented as the mean $\pm \mathrm{SD}$. $\left(\mathrm{N}=5\right.$ for each group; $\left.{ }^{*} \mathrm{p}<0.05\right)$. BV/TV: bone volume/ tissue volume; Tb.Th: trabecular thickness. (D) The average weight of rats in each group was measured and expressed as the mean $\pm \mathrm{SD}$. ( $\mathrm{N}=10$ for each group).

Animal model of alcohol-induced ONFH was alleviated by cordycepin

To investigate the effects of cordycepin on ethanol-induced ONFH, the rat model of ONFH was established by a Lieber-Decarli ethanol-containing liquid diet $[23,24]$, followed

\section{KARGER}




\section{Cellular Physiology Cell Physiol Biochem 2017;42:2391-2403 and Biochemistry \begin{tabular}{l|l} 
DOI: 10.1159/000480181 2017 The Author(s). Published by S. Karger AG, Basel \\
Publisher
\end{tabular} \\ Chen et al.: Protective Effect of Cordycepin On Alcohol-Induced ONFH}

by intraperitoneal injection of cordycepin or an equal volume of saline as control. Six weeks after corresponding treatment, micro-CT scanning was performed for evaluation and quantification of bone tissues of the femoral head. Seven of the 10 rats in the AL group showed visible signs of osteonecrosis, while only 2 of the 10 rats in the AL+ cordycepin group showed mild ONFH (Fig. 4A). No rat was found with osteonecrosis in the NC group. Quantitative analyses of micro-CT data further confirmed the effect of alcohol and the efficacy of cordycepin on preventing alcohol-induced ONFH in rats. As shown in Fig. 4B-4D, the BV/ TV ratio (23.54 \pm 9.42$)$ and Tb.Th $(0.0722 \pm 0.004)$ in the AL group were significantly reduced compared to those in the NC group (63.99 \pm 5.06 and $0.156 \pm 0.029$, respectively; both $p<$ $0.05)$. Supplementation with cordycepin significantly increased the BV/TV ratio $(44.42 \pm$ $14.45, p<0.05$ compared to the AL group) and Tb.Th $(0.117 \pm 0.045, p<0.05$ compared to the AL group).

Next, histopathological examinations were carried out to verify our micro-CT findings. We identified typical osteonecrotic lesions of the femoral head in 7/10 rats in the AL group, as diffuse empty lacunae present in bone trabeculae, surrounded by bone marrow cell necrosis [24-26]. After six weeks of corresponding treatment, the subchondral area of femoral heads in the AL group ( $7 / 10$ rats) were found occupied by empty lacunae with surrounding bone

Fig. 5. The alcohol-induced ONFH was alleviated by cordycepin. (A) H\&E staining of the femoral head revealed obvious osteonecrosis in the AL group. Empty lacunae in subchondral trabeculae (black triangles) with surrounding necrosis of bone marrow cells (black star) were present in the AL group, while only few empty lacunae were detected in the AL+ cordycepin group. (B) TUNEL staining of the specimen indicated increased apoptosis in the AL group, and cellular apoptosis was alleviated by cordycepin administration. The TUNEL positive cells (green triangles), karyopyknosis and nuclear fragmentation (black triangles) were increased in the AL group but were decreased in the AL+ cordycepin group. (C) Quantification of TUNEL-positive cells in each group were calculated $(\mathrm{N}=3$ for each group; ${ }^{*}$ < 0.05 ).

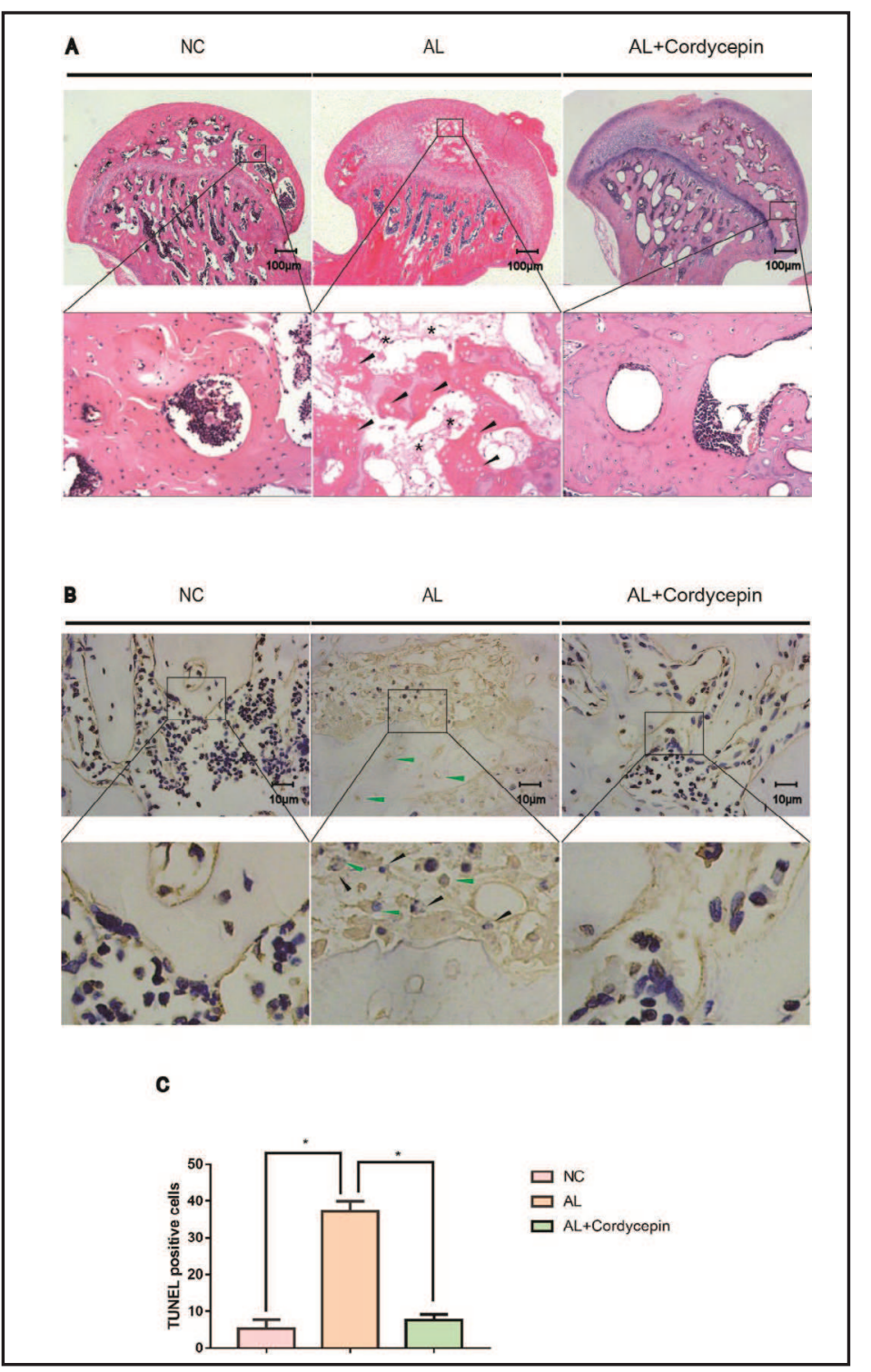




\section{Cellular Physiology Cell Physiol Biochem 2017;42:2391-2403 and Biochemistry DOI: 10.1159/000480181 2017 1 () 2017 The Author(s). Published by S. Karger AG, Basel}

Chen et al.: Protective Effect of Cordycepin On Alcohol-Induced ONFH

marrow cell necrosis in H\&E images (Fig. 5A). However, significantly fewer pathological changes were observed in the AL+ cordycepin group, while no apparent histopathological changes were found in the NC group throughout the study (Fig. 5A). Those data supported our micro-CT results of the protective effect of cordycepin on alcohol-induced ONFH.

Furthermore, apoptosis is regarded as one of the most crucial changes in the pathological process of ONFH $[10,27]$. We carried out TUNEL staining of femoral head specimens to detect apoptosis. As shown in Fig. 5B, significant TUNEL positive osteocytes (green triangles) and the remaining empty lacunae were observed in the trabecular bone of the AL group compared to that in the control (Fig. 5B). We also noticed karyopyknosis and nuclear fragmentation (black triangles) in the marrow cavity, surrounded by bone marrow cell necrosis, which was typical of osteonecrotic lesions of the femoral head. However, these pathological changes were reduced by cordycepin administration as indicated in Fig. 5B. Quantification of the image results is shown in Fig. $5 \mathrm{C}\left({ }^{*} p<0.05\right)$. All three groups of rats were healthy throughout the study and without significant differences in body weight (Fig. 4D).

\section{Alcohol-suppressing osteogenic activity was rescued by cordycepin in vivo}

We performed histochemical staining of osteogenesis-related markers, including OCN and COL1, of specimens from rats' femoral heads. The results showed less OCN- and COL1positive staining on subchondral trabeculae in the AL group compared with that in the NC

Fig. 6. Alcohol-induced osteogenic suppression was rescued by cordycepin in vivo. (A) Immunohistochemical staining for COL1. Fewer trabeculae (black stars) were positive for COL1 in the AL group, while more COL1-positive trabeculae were observed in the $\mathrm{AL}+$ cordycepin group. (B) Immunohistochemical staining for OCN. Less positive staining of OCN (red stars) in the femoral heads of the AL group, whereas increased OCN-positive trabeculae were observed in the AL+ cordycepin group. (C-D) Quantification of positive staining area in each group were calculated $(\mathrm{N}=3$ for each group; ${ }^{*} \mathrm{p}<0.05$ ).

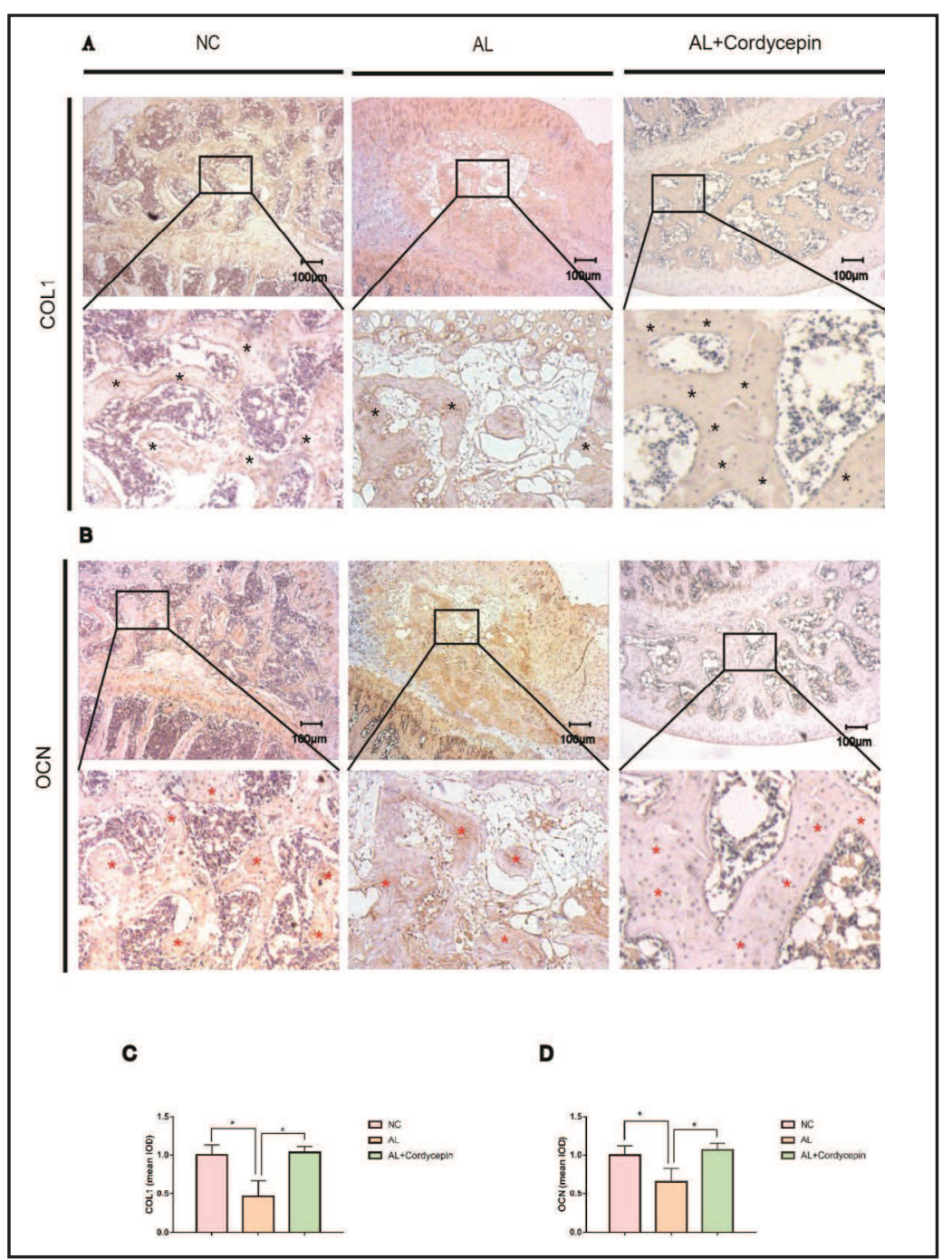




\section{Cellular Physiology Cell Physiol Biochem 2017;42:2391-2403

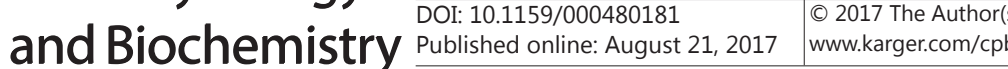 \\ Chen et al.: Protective Effect of Cordycepin On Alcohol-Induced ONFH}

group (Fig. 6A-B), whereas co-administration of cordycepin markedly increased the positive staining compared with that in the AL group (Fig. 6A-D). These results were consistent with previous in vitro investigations, namely, that cordycepin played an osteo-protective role on ethanol-treated BMSCs. Taken together, our in vivo data demonstrated the enhancing effect of cordycepin on bone tissue regeneration and resistance to counteract ethanol's effect and thereby protect the femoral head against necrosis.

\section{Discussion}

ONFH is a common deteriorating complication that ultimately results in hip disability. Invasive surgery for ONFH includes hip replacement, core decompression and rotational osteotomy [28-31]. The detailed pathogenic process of ONFH has not been well established. Alcohol has been demonstrated as one of the most important risk factors of atraumatic $\mathrm{ONFH}$, apart from other risk factors including glucocorticoids, lipid metabolism disorders, thrombophilia and hypofibrinolysis [32-34]. Although numerous therapeutic attempts have been made for the non-surgical treatment of ONFH to prevent the need for corrective procedures, there is still room for improvement. In our study, we confirmed for the first time that cordycepin could reduce the ethanol-induced suppressive effect on hBMSCs and prevent the development of alcohol-induced ONFH in a rat model.

In the present study, we demonstrated that cordycepin reversed the inhibitory effects of ethanol on the osteogenic differentiation of hBMSCs and prevented the development of alcohol-induced ONFH in a rat model. Alcohol-induced ONFH was induced by a LieberDecarli alcohol-containing diet [24]. The in vivo results revealed that cordycepin reduced pathological changes including the decrease in subchondral trabecular bone, diffuse empty lacunae in trabeculae bone and surrounding bone marrow necrosis in typical alcohol-induced ONFH [24]. Current studies support that apoptosis is involved in the progression of ONFH $[27,35]$. Data from the TUNEL assay showed the protective effects of cordycepin against the alcohol-induced pro-apoptosis in rat femoral heads. Previous studies suggested that decreased osteogenic activity in an animal body was closely related with the development of ONFH [36-38]. Therefore, using micro-CT analyses, we found that the bone mineral density and numbers of trabecular bones were decreased in the AL group but were reversed in the $\mathrm{AL}+$ cordycepin group. Immunohistochemical observation of OCN and COL1 staining further visualized that alcohol consumption decreased the osteogenic activity in femoral heads, while co-administration of cordycepin enhanced osteogenesis. In vitro, the results of ALP activity, mRNA expressions of OCN and BMP2 demonstrated that $10 \mu \mathrm{g} / \mathrm{ml}$ of cordycepin was sufficient to rescue the ethanol-induced suppression of osteogenesis in hBMSCs. It was noted that the concentration of $10 \mu \mathrm{g} / \mathrm{ml}$ cordycepin did not suppress the proliferation of hBMSCs. Further, the osteo-enhancing effect of cordycepin was confirmed and visualized by western blots and immunofluorescence. During the in vivo experiments, all rats were healthy and no changes in the body weight, appearance, and behavior of the rats were observed.

Cordycepin is the main effective component extracted from the traditional Chinese medicine cordyceps militaris. For many years, cordycepin has been applied for various medical purposes, including anti-oxidative stress [39], enhancing bone mineral density [18], preventing myocardial ischemia/reperfusion injury [40], alleviating alcoholic hepatotoxicity [41] and protecting neurons against toxic agents [42]. However, whether cordycepin could exert sufficient protective effects on alcohol-induced ONFH has not been determined. A study by Wang et al [43]. supported that treatment with cordycepin in BMSCs activated the Wnt/ $\beta$-catenin pathway, exerting an anti-oxidative property on bone metabolism $[39,42]$ and enhancing osteogenic differentiation. Recent studies support that the Wnt signaling pathway plays an important role in osteogenic differentiation and bone formation $[44,45]$. Activation of canonical Wnt signaling results in higher bone density, whereas disruption of its activation impairs osteogenesis [46]. Wnt signaling pathway contributes to osteogenesis primarily through activation of $\beta$-catenin, which plays the central role in the initiation of 


\section{Cellular Physiology Cell Physiol Biochem 2017;42:2391-2403

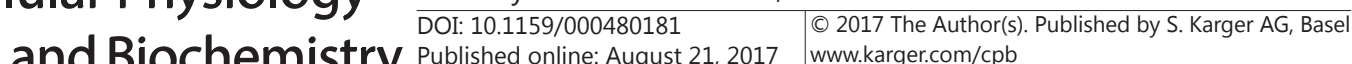 \\ Chen et al.: Protective Effect of Cordycepin On Alcohol-Induced ONFH}

osteogenesis [47]. Chen et al. reported that ethanol suppresses the Wnt/ $\beta$-catenin pathway in BMSCs and retards the osteogenic activity [21]. Herein, we assume that the Wnt/ $\beta$-catenin signaling pathway might be involved in osteo-protective effect of cordycepin on ethanolinduced anti-osteogenic effects in hBMSCs. We performed western blots of $\beta$-catenin in hBMSCs. The result showed significantly decreased $\beta$-catenin hBMSCs by ethanol treatment, which was similar to previous studies. However, $1 \mu \mathrm{g} / \mathrm{ml}$ and $10 \mu \mathrm{g} / \mathrm{ml}$ cordycepin reversed this blockage effect. It was noted that the change in $\beta$-catenin level was paralleled with the Runx2 level, which is the main transcription factor of osteogenic differentiation. These findings confirmed our assumptions that the Wnt/ $\beta$-catenin pathway is associated with the suppression of osteogenic differentiation by ethanol in hBMSCs, which is re-activated by cordycepin.

In addition, cordycepin appears to be a safe reagent. All rats were in good health in the animal experiments. No significant difference in body weight or behavior was detected between groups throughout the study.

\section{Conclusion}

In conclusion, our study demonstrated the protective effect of cordycepin against alcohol-induced ONFH both in vitro and in vivo. Cordycepin exerts this protective effect partially by enhancing the osteogenic differentiation of BMSCs associated with the Wnt/ $\beta$ catenin pathway. Therefore, cordycepin may serve as a valuable therapeutic strategy for alcohol-induced ONFH in humans.

\section{Acknowledgements}

The current research was supported in part by the National Natural Science Foundation of China (grant no. 81672143).

\section{Disclosure Statement}

The authors declare no competing financial interests.

\section{References}

1 Mikosch P: Alcohol and bone. Wien Med Wochenschr 2014;164:15-24.

2 Li J, Wang Y, Li Y, Sun J, Zhao G: The effect of combined regulation of the expression of peroxisome proliferator-activated receptor-gamma and calcitonin gene-related peptide on alcohol-induced adipogenic differentiation of bone marrow mesenchymal stem cells. Mol Cell Biochem 2014;392:39-48.

3 Wang YS, Zhang Y, Li JW, Yang GH, Li JF, Yang J, Yang GH: A modified technique of bone grafting pedicled with femoral quadratus for alcohol-induced osteonecrosis of the femoral head. Chin Med J 2010;123:28472852.

4 Ikeuchi K, Hasegawa Y, Seki T, Takegami Y, Amano T, Ishiguro N: Epidemiology of nontraumatic osteonecrosis of the femoral head in Japan. Mod Rheumatol 2015;25:278-281.

-5 Fukushima W, Fujioka M, Kubo T, Tamakoshi A, Nagai M, Hirota Y: Nationwide epidemiologic survey of idiopathic osteonecrosis of the femoral head. Clin Orthop Relat Res 2010;468:2715-2724.

6 Santori C, Ceccanti M, Diacinti D, Attilia ML, Toppo L, D’Erasmo E, Romagnoli E, Mascia ML, Cipriani C, Prastaro A, Carnevale V, Minisola S: Skeletal turnover, bone mineral density, and fractures in male chronic abusers of alcohol. J Endocrinol Invest 2008;31:321-326.

7 Maran A, Zhang M, Spelsberg TC, Turner RT: The dose-response effects of ethanol on the human fetal osteoblastic cell line. J Bone Miner Res 2001;16:270-276. 


\section{Cellular Physiology Cell Physiol Biochem 2017;42:2391-2403 \begin{tabular}{l|l|l} 
DOI: 10.1159/000480181 21,2017 & $\begin{array}{l}\text { ( ) 2017 The Author(s). Published by S. Karger AG, Basel } \\
\text { www.karger.com/cpb }\end{array}$
\end{tabular}}

Chen et al.: Protective Effect of Cordycepin On Alcohol-Induced ONFH

-8 Shankar K, Hidestrand M, Liu X, Chen JR, Haley R, Perrien DS, Skinner RA, Lumpkin CK Jr, Badger TM, Ronis MJ: Chronic ethanol consumption inhibits postlactational anabolic bone rebuilding in female rats. J Bone Miner Res 2008;23:338-349.

-9 Trevisiol CH, Turner RT, Pfaff JE, Hunter JC, Menagh PJ, Hardin K, Ho E, Iwaniec UT: Impaired osteoinduction in a rat model for chronic alcohol abuse. Bone 2007;41:175-180.

10 Maurel DB, Jaffre C, Rochefort GY, Aveline PC, Boisseau N, Uzbekov R, Gosset D, Pichon C, Fazzalari NL, Pallu S, Benhamou CL: Low bone accrual is associated with osteocyte apoptosis in alcohol-induced osteopenia. Bone 2011;49:543-552.

-11 Aicher WK, Buhring HJ, Hart M, Rolauffs B, Badke A, Klein G: Regeneration of cartilage and bone by defined subsets of mesenchymal stromal cells--potential and pitfalls. Adv Drug Deliv Rev 2011;63:342-351.

12 Pontikoglou C, Deschaseaux F, Sensebe L, Papadaki HA: Bone marrow mesenchymal stem cells: biological properties and their role in hematopoiesis and hematopoietic stem cell transplantation. Stem Cell Rev 2011;7:569-589.

13 Kim IG, Hwang MP, Du P, Ko J, Ha CW, Do SH, Park K: Bioactive cell-derived matrices combined with polymer mesh scaffold for osteogenesis and bone healing. Biomaterials 2015;50:75-86.

14 Driver J, Weber CE, Callaci JJ, Kothari AN, Zapf MA, Roper PM, Borys D, Franzen CA, Gupta GN, Wai PY, Zhang J, Denning MF, Kuo PC, Mi Z: Alcohol inhibits osteopontin-dependent transforming growth factor-beta1 expression in human mesenchymal stem cells. J Biol Chem 2015;290:9959-9973.

15 Sun ZB, Wang JW, Xiao H, Zhang QS, Kan WS, Mo FB, Hu S, Ye SN: Icariin may benefit the mesenchymal stem cells of patients with steroid-associated osteonecrosis by ABCB1-promoter demethylation: a preliminary study. Osteoporos Int 2015;26:187-197.

-16 Gu C, Xu Y, Zhang S, Guan H, Song S, Wang X, Wang Y, Li Y, Zhao G: miR-27a attenuates adipogenesis and promotes osteogenesis in steroid-induced rat BMSCs by targeting PPARgamma and GREM1. Sci Rep 2016;6:38491.

17 Ng TB, Wang HX: Pharmacological actions of Cordyceps, a prized folk medicine. J Pharm Pharmacol 2005;57:1509-1519.

18 Dou C, Cao Z, Ding N, Hou T, Luo F, Kang F, Yang X, Jiang H, Xie Z, Hu M, Xu J, Dong S: Cordycepin prevents bone loss through inhibiting osteoclastogenesis by scavenging ROS generation. Nutrients 2016;8:231.

19 Kim J, Lee H, Kang KS, Chun KH, Hwang GS: Cordyceps militaris mushroom and cordycepin inhibit RANKLinduced osteoclast differentiation. J Med Food 2015;18:446-452.

-20 Zhang DW, Deng H, Qi W, Zhao GY, Cao XR: Osteoprotective effect of cordycepin on estrogen deficiencyinduced osteoporosis in vitro and in vivo. BioMed Res Int 2015;2015:423869.

-21 Chen JR, Lazarenko OP, Shankar K, Blackburn ML, Badger TM, Ronis MJ: A role for ethanol-induced oxidative stress in controlling lineage commitment of mesenchymal stromal cells through inhibition of Wnt/beta-catenin signaling. J Bone Miner Res 2010;25:1117-1127.

22 Chen YX, Tao SC, Xu ZL, Yin WJ, Zhang YL, Yin JH, Gao YS, Zhang CQ: Novel Akt activator SC-79 is a potential treatment for alcohol-induced osteonecrosis of the femoral head. Oncotarget 2017;8:31065-31078.

-23 Lieber CS, DeCarli LM: The feeding of alcohol in liquid diets: two decades of applications and 1982 update. Alcohol Clin Exp Res 1982;6:523-531.

24 Okazaki S, Nagoya S, Tateda K, Katada R, Mizuo K, Watanabe S, Yamashita T, Matsumoto H: Experimental rat model for alcohol-induced osteonecrosis of the femoral head. Int J Exp Pathol 2013;94:312-319.

-25 Ichiseki T, Kaneuji A, Ueda Y, Nakagawa S, Mikami T, Fukui K, Matsumoto T: Osteonecrosis development in a novel rat model characterized by a single application of oxidative stress. Arthritis Rheumatism 2011;63:2138-2141.

-26 Okazaki S, Nishitani Y, Nagoya S, Kaya M, Yamashita T, Matsumoto H: Femoral head osteonecrosis can be caused by disruption of the systemic immune response via the toll-like receptor 4 signalling pathway. Rheumatology (Oxford) 2009;48:227-232.

-27 Ko JY, Wang FS, Wang CJ, Wong T, Chou WY, Tseng SL: Increased Dickkopf-1 expression accelerates bone cell apoptosis in femoral head osteonecrosis. Bone 2010;46:584-591.

-28 Kubo Y, Yamamoto T: Patient-reported outcomes of femoral osteotomy and total hip arthroplasty for osteonecrosis of the femoral head: a prospective case series study. Springerplus 2016;5:1880.

29 Zhao DW, Yu XB: Core decompression treatment of early-stage osteonecrosis of femoral head resulted from venous stasis or artery blood supply insufficiency. J Surg Res 2015;194:614-621. 


\section{Cellular Physiology \\ Cell Physiol Biochem 2017;42:2391-2403 and Biochemistry

-30 Kubo T, Ueshima K, Saito M, Ishida M, Arai Y, Fujiwara H: Clinical and basic research on steroid-induced osteonecrosis of the femoral head in Japan. J Orthop Sci 2016;21:407-413.

-31 Motomura G, Yamamoto T, Suenaga K, Nakashima Y, Mawatari T, Ikemura S, Iwamoto Y: Long-term outcome of transtrochanteric anterior rotational osteotomy for osteonecrosis of the femoral head in patients with systemic lupus erythematosus. Lupus 2010;19:860-865.

-32 Baek SH, Kim KI, Yoon KS, Kim TH, Kim SY: Genome-wide association scans for idiopathic osteonecrosis of the femoral head in a Korean population. Mol Med Rep 2017;15:750-758.

-33 Gagala J, Buraczynska M, Mazurkiewicz T, Ksiazek A: Prevalence of genetic risk factors related with thrombophilia and hypofibrinolysis in patients with osteonecrosis of the femoral head in Poland. BMC Musculoskelet Disord 2013;14:264.

-34 Cui L, Zhuang Q, Lin J, Jin J, Zhang K, Cao L, Lin J, Yan S, Guo W, He W, Pei F, Zhou Y, Weng X: Multicentric epidemiologic study on six thousand three hundred and ninety five cases of femoral head osteonecrosis in China. Int Orthop 2016;40:267-276.

-35 Zheng H, Yang E, Peng H, Li J, Chen S, Zhou J, Fang H, Qiu B, Wang Z: Gastrodin prevents steroid-induced osteonecrosis of the femoral head in rats by anti-apoptosis. Chin Med J 2014;127:3926-3931.

-36 Yu Z, Fan L, Li J, Ge Z, Dang X, Wang K: Lithium prevents rat steroid-related osteonecrosis of the femoral head by beta-catenin activation. Endocrine 2016;52:380-390.

-37 Zhang YL, Yin JH, Ding H, Zhang W, Zhang CQ Gao YS: Vitamin K2 prevents glucocorticoid-induced osteonecrosis of the femoral head in rats. Int J Biol Sci 2016;12:347-358.

38 Hao C, Yang S, Xu W, Shen JK, Ye S, Liu X, Dong Z, Xiao B, Feng Y: MiR-708 promotes steroid-induced osteonecrosis of femoral head, suppresses osteogenic differentiation by targeting SMAD3. Sci Rep 2016;6:22599.

-39 Ramesh T, Yoo SK, Kim SW, Hwang SY, Sohn SH, Kim IW, Kim SK: Cordycepin (3'-deoxyadenosine) attenuates age-related oxidative stress and ameliorates antioxidant capacity in rats. Exp Gerontol 2012;47:979-987.

40 Park ES, Kang DH, Yang MK, Kang JC, Jang YC, Park JS, Kim SK, Shin HS: Cordycepin, 3'-deoxyadenosine, prevents rat hearts from ischemia/reperfusion injury via activation of Akt/GSK-3beta/p70S6K signaling pathway and HO-1 expression. Cardiovasc Toxicol 2014;14:1-9.

41 Cha JY, Ahn HY, Cho YS, Je JY: Protective effect of cordycepin-enriched Cordyceps militaris on alcoholic hepatotoxicity in Sprague-Dawley rats. Food Chem Toxicol 2013;60:52-57.

-42 Olatunji OJ, Feng Y, Olatunji 00, Tang J, Ouyang Z, Su Z: Cordycepin protects PC12 cells against 6-hydroxydopamine induced neurotoxicity via its antioxidant properties. Biomed Pharmacother 2016;81:7-14.

43 Wang F, Yin P, Lu Y, Zhou Z, Jiang C, Liu Y, Yu X: Cordycepin prevents oxidative stress-induced inhibition of osteogenesis. Oncotarget 2015;6:35496-35508.

44 Rudnicki MA, Williams BO: Wnt signaling in bone and muscle. Bone 2015;80:60-66.

45 Lerner UH, Ohlsson C: The WNT system: background and its role in bone. J Intern Med 2015;277:630-649.

-46 Georgiou KR, King TJ, Scherer MA, Zhou H, Foster BK, Xian CJ: Attenuated Wnt/beta-catenin signalling mediates methotrexate chemotherapy-induced bone loss and marrow adiposity in rats. Bone 2012;50:1223-1233.

47 Duan P, Bonewald LF: The role of the wnt/beta-catenin signaling pathway in formation and maintenance of bone and teeth. Int J Biochem Cell Biol 2016;77:23-29. 